\title{
Solid-phase microextraction for bioconcentration studies according to OECD TG 305
}

\author{
Rolf-Alexander Düring ${ }^{1 *}$, Leonard Böhm ${ }^{1}$ and Christian Schlechtriem²
}

\begin{abstract}
An important aim of the European Community Regulation on chemicals and their safe use is the identification of (very) persistent, (very) bioaccumulative, and toxic substances. In other regulatory chemical safety assessments (pharmaceuticals, biocides, pesticides), the identification of such (very) persistent, (very) bioaccumulative, and toxic substances is of increasing importance. Solid-phase microextraction is especially capable of extracting total water concentrations as well as the freely dissolved fraction of analytes in the water phase, which is available for bioconcentration in fish. However, although already well established in environmental analyses to determine and quantify analytes mainly in aqueous matrices, solid-phase microextraction is still a rather unusual method in regulatory ecotoxicological research. Here, the potential benefits and drawbacks of solid-phase microextraction are discussed as an analytical routine approach for aquatic bioconcentration studies according to OECD TG 305, with a special focus on the testing of hydrophobic organic compounds characterized by log $K_{\text {ow }}>5$.
\end{abstract}

Keywords: particulate and dissolved organic matter, bioconcentration factor, bioavailability, hydrophobic organic chemicals, registration and authorization of chemicals

\section{Background}

The European Community Regulation on chemicals and their safe use dealing with Registration, Evaluation, Authorisation and Restriction of Chemicals [REACh] [1] defines specific registration requirements for substances that are (very) persistent, (very) bioaccumulative, and toxic [PBT/vPvB substances]. As part of other regulatory chemical safety assessments such as for pharmaceuticals, biocides, and pesticides, the identification of $\mathrm{PBT} / \mathrm{vPvB}$ substances is of increasing importance. With regard to the bioaccumulative properties of substances, bioconcentration factors $[\mathrm{BCF}]$, which are defined as the concentration of a test substance in fish divided by the concentration of the chemical in the surrounding medium exceeding 2,000 and 5,000, are limiting criteria for $\mathrm{B}$ and $\mathrm{vB}$ substances, respectively. As a consequence, numerous BCF tests for hydrophobic organic compounds [HOCs] must be performed according to REACh requirements, and thus, efficient and reliable analytical methods for water and tissue samples are needed to guarantee accurate BCF estimates

\footnotetext{
* Correspondence: rolf-alexander.duering@umwelt.uni-giessen.de ${ }^{1}$ Institute of Soil Science and Soil Conservation, Research Centre for BioSystems, Land Use and Nutrition (IFZ) Justus Liebig University Giessen, Heinrich-Buff-Ring 26-32, Giessen, 35392, Germany Full list of author information is available at the end of the article
}

from flow-through fish tests carried out according to Organisation for Economic Co-operation and Development Technical Guideline [OECD TG] 305 [2].

OECD TG 305 describes a procedure for characterizing the bioconcentration potential of substances in fish. The test consists of two phases: the exposure (uptake) and post-exposure (depuration) phases. During the uptake phase, separate groups of fish of one species are exposed to at least two concentrations of the test substance. They are then transferred to a medium free of the test substance for the depuration phase. The concentration of the test substance in the fish is followed through both phases of the test. Where possible, the BCF is calculated preferably both as the ratio of the concentration in the fish and in the water at apparent steady state $\left(\mathrm{BCF}_{\mathrm{ss}}\right)$ and as the kinetic bioconcentration factor $\mathrm{BCF}_{\mathrm{K}}$, which is the ratio of the rate constants of uptake $\left(k_{1}\right)$ and depuration $\left(k_{2}\right)$ assuming first-order kinetics [2]. OECD TG 305 does not define specific analytical techniques to determine analyte concentrations in the water phase. Commonly applied are liquid-liquid extraction [LLE] and solid-phase extraction [SPE].

LLE can yield exhaustive extraction of the analytes but is time-consuming, is labor-intensive, and requires the

\section{Springer}


use of considerable amounts of harmful solvents. In contrast, SPE has emerged as a powerful and solventsaving tool to extract and purify analytes and now plays an important role in a broad range of applications [3]. Compared with LLE, SPE offers reduced processing time and significant solvent saving. However, this method requires multi-steps and is still time-consuming due to limited flow velocity during sample concentration and elution steps. Both LLE and SPE are disadvantageous in terms of losses in the evaporation step. However, higher concentrations can occur due to risks of contamination.

Solid-phase microextraction [SPME] combines selective extraction and enrichment of analytes from liquid, gaseous, or solid samples with sample introduction to the analytical device. For HOCs, SPME is preferably coupled to gas chromatography [GC], with a direct thermodesorption of the analytes in the injection system. First reported in 1990, SPME provides solventless extraction of water samples and minimizes problems such as volatilization losses and contamination [4].

The principle of SPME is the partitioning of analytes from the sample to a coated fiber in the course of an equilibration. This process is highly dependent on the characteristics of the analyte, the sample matrix, and the ambient conditions. Background and applications of SPME have been summarized in textbooks and reviews [4-7].

SPME has been applied in many different areas such as environmental analysis where precise quantification must be validated. This technique has been shown to be suitable and valid for a wide range of analytes from volatile to nonvolatile compounds including many HOCs [8-11] among others. As a consequence, a German DIN standard operational procedure for the analysis of 22 pesticides of different polarities from water samples was established in 2007 [12]. With regard to this standard procedure, SPME can be seen as a well-established and accepted method for the quantitative analysis of organic contaminants from moderate to high hydrophobicity in water samples.

Within bioconcentration tests, it was observed that highly HOCs with $\log K_{\mathrm{OW}}>5$ to 6 bioconcentrate less than what may be expected from their hydrophobicity. Explanations for this hydrophobicity 'cutoff' might be a reduced membrane permeation caused by steric effects of larger molecules or measurement artifacts [13], possibly because of the presence of organic matter [OM] in the test system. The influence of OM, quantified by the total organic carbon [TOC] in the test system on BCF values, has been noted since the 1980s because the bioconcentration of a substance is mainly influenced by its bioavailability; that is, differentiated from a substance's total amount, only molecules that are bioavailable can cross an organism's cellular membrane from the medium the organism inhabits at a given time [14]. Increased concentrations of $\mathrm{OM}$ in the aquatic phase may reduce bioavailability and calculated BCF values when using total aqueous analyte concentrations [15]. Therefore, the OECD TG 305 requires tanks to be kept as clean as possible and to monitor the TOC content throughout the experiment. Particulate matter and TOC are accepted up to 5 and $2 \mathrm{mg} \mathrm{L}^{-1}$ in the dilution water, respectively, and the concentration should not exceed $10 \mathrm{mg} \mathrm{L}^{-1} \mathrm{TOC}$ in the test vessels (without TOC from test substances and solubilizing agents). However, HOCs bind to OM far below a TOC content of $10 \mathrm{mg} \mathrm{L}^{-1}$ [16]. While the determination of freely dissolved concentrations has long been known to be laborious, SPME is suitable to distinguish freely dissolved and total concentrations and seems to be useful for various environmental and (eco)toxicological investigations [17]. Nevertheless, many articles dealing with the measurement of freely dissolved concentrations by SPME use it as a passive sampling system and not as an analytical routine method. If SPME is considered as an alternative routine method, directly coupled to GC, equilibrium conditions and solvent extraction can be disregarded.

Here, we discuss the suitability of SPME in fish bioconcentration studies by presenting its benefits and drawbacks. Recommendations for this application and further research needs are provided.

\section{SPME: benefits and drawbacks in work routine}

Since the equilibration of the analyte between the matrix and the fiber can take more than a day, it is recommended to stop the equilibration process in the dynamic range, which is possible if ambient conditions are held constant. Here, it is essential that temperature, extraction time, and stirring are identical among samples and standard solutions, which makes an autosampler with SPME device and agitator (well-defined shaking and heating) indispensable. To maintain reproducibility, extraction parameters such as ion strength and $\mathrm{pH}$ of the water solution must be consistent during a series of analyses [4]. Especially for HOCs, where thermodesorption from the SPME fiber can be insufficient following the GC measurement, an additional bakeout oven is necessary.

Measuring total analyte concentrations by an external standard calibration is well suited for homogeneous aqueous samples with minor interference. Minor errors in the process, the continuous decrease of fiber extraction efficiency as well as the potential variation in instrument sensitivity can be eliminated by the use of internal standards. Accordingly, sample-to-sample variations in the extraction and desorption efficiencies caused by the sample matrix, i.e., due to the presence of OM, can be compensated $[8,10,18]$. If this compensation cannot be assured, standard addition is an accurate method to use for quantification though still time-consuming. For both internal standard and standard addition methods, interaction of the matrix 
and both analytes and internal standards must be considered when quantifying the total analyte concentration.

If coupled to GC, SPME generally has a high sensitivity for HOCs and quantification is possible at trace levels, allowing for studies with low concentrations of test substances. If required, small sample volumes of 5 to $20 \mathrm{~mL}$ can be handled. The quantification of multiple analytes in water has been successfully applied for some years [10-12]. Validated and official standard protocols already exist; however, preliminary studies should always be carried out to assess extraction kinetics and time, as well as desorption temperature, to optimize extraction conditions for new analytes.

Analysis of total analyte concentrations yields comparable results for LLE and SPME [8]. However, SPME is a quicker approach with lower current costs due to reduced time for preparation, the reusability of SPME fibers, and waiving of solvents, which enhances SPME as a more environmentally friendly approach.

In contrast to conventional LLE, where a prepared small sample extract can be measured several times, for SPME analysis, an aliquot of the original sample is measured, which requires retained water samples for repetitive measurements. Due to the higher diameter of the hollow needle of an SPME fiber, as compared with syringe needles used for the injection of liquid extracts, the injection system and especially the septum require special attention as otherwise a septum-free injection system must be chosen.

\section{SPME: potential and limitations}

Transport behavior, bioavailability, and toxicity of organic chemicals are heavily dependent on their freely available concentration $[17,19]$. However, HOCs with octanol-water coefficients $\left[K_{\mathrm{OW}}\right]>10^{5}$ show a high propensity to bind to $\mathrm{OM}$. If binding to $\mathrm{OM}$ is not taken into consideration, the quantitative description of dose-response relationships might thus be incorrect. Therefore, it is necessary to distinguish between the total and the freely dissolved fraction of hydrophobic test substances, both present in the water phase.

With LLE, freely dissolved fractions are not distinguished from bound amounts of the analytes in more complex water samples (i.e., water samples with particulate and dissolved OM). The separation with SPE is generally possible but fails due to the high uncertainty of the method, especially when free amounts of highly HOCs are to be quantified [20].

Apart from containing total amounts, analysis by SPME provides information on the freely dissolved analyte concentrations. The SPME technique can be used to determine the partitioning of HOCs in a three-phase water system consisting of a solution, dissolved organic matter, and particulate organic matter and allows for the estimation of specific partition coefficients such as $K_{\mathrm{OC}}$ and
$K_{\text {DOC }}[16,21,22]$. Taking this advantage, SPME may help to determine more accurate BCFs of organic chemicals because only the freely dissolved fraction partitions to the SPME fiber [23].

While SPME is often described for the measurement of freely dissolved concentrations, different manual approaches are used to extract analytes from water, soils, or sediments for in situ measurements or as a substitute for test organisms. For this, pieces of fiber coated with polydimethylsiloxane [PDMS] are equilibrated in the sample and afterwards desorbed thermally or by solvents [13,23-27]. PDMS is the most common fiber coating for the analysis of HOCs.

Nevertheless, automated SPME also works for measuring free concentrations, solventless at pre-equilibrium conditions. Small aliquots $(\leq 20 \mathrm{~mL})$ of water samples are filled in SPME vials, tightly closed, and measured subsequently without any additional manual treatment. However, no internal standards can be used to correct variations during the measuring process, which is challenging when working with mass spectrometry [MS]. Internal standards cannot be used due to their similarity to the analyte. For that reason, the internal standards are also bound by OM, and a correction by internal standards would not lead to the freely dissolved concentration. However, the absence of internal standards is also a drawback in manual SPME approaches. Without internal standardization, ageing of the SPME fiber is challenging, as is the variation of the MS performance. Since a correction method for routine analysis is missing for MS, the alternative is to measure freely dissolved concentrations with more robust detection systems such as GC coupled to a flame ionization detector or an electron capture detector. In addition, due to the fast process, SPME seems capable to easily monitor the course of a BCF study with high temporal resolution.

\section{The use of SPME in fish bioconcentration studies: conclusions and recommendations}

SPME coupled to GC is a well-established method in water analysis, especially for total analyte concentrations, and seems capable of automatically measuring analyte concentrations in the water phase in standardized bioconcentration studies. However, with few limitations, automated SPME allows the gain of free analyte concentrations simultaneously. With regard to the partitioning of HOCs in aquatic systems, it can be expected that the conventional extraction procedure (LLE) may capture significant amounts of molecules bound to suspended and dissolved OM that are not available to fish. These amounts do not contribute to BCF but are extracted by the classical total extraction techniques. Aqueous analyte concentrations with special reference to different contents of TOC were investigated [28]. These first results showed that the freely dissolved concentration of highly 
HOCs can be significantly reduced due to sorption to natural OM - already beneath the permitted concentration of $2 \mathrm{mg} \mathrm{L}^{-1}$ TOC in the dilution water according to OECD TG 305. It is to be expected that resulting BCF values for highly HOCs may be inaccurate and lead to an underestimation of BCFs. This aspect was raised by Jonker and van der Heijden [13], although their study on the determination of BCFs was not carried out with fish and without measuring of TOC concentrations. To examine if there is an underestimation of fish BCFs caused by measuring artifacts, flow-through fish tests with HOCs should be carried out under realistic conditions. Likewise, the influence of different organic carbon levels and the influence of OM quality in experimental systems should be investigated in-depth. Based on the approaches and studies described here, it will be possible to further elucidate whether highly HOCs with $\log K_{\mathrm{OW}}$ values $>5$ bioconcentrate less than what may be expected from their hydrophobicity.

The OECD TG 305 is currently under revision. SPME should be recommended for BCF studies on highly HOCs to obtain information on the ratio between bound and freely dissolved compounds. Internal standard-corrected results obtained by SPME and LLE are presumably equal; therefore, the results of former and future BCF studies should be comparable even if LLE and SPME are used in parallel for the extraction of total water concentrations. For highly HOCs, SPME can be beneficial for simultaneously gaining free concentrations. However, an equivalency of SPME and LLE in BCF studies should be further studied to guarantee their use in a regulatory context.

Variation of results between SPME and LLE will probably be lower than variations of (LLE) results without precisely specified TOC contents. Unfortunately, TOC values are often missing, even though declaring the TOC values is indispensable for a correct interpretation and classification of BCFs [15].

For highly HOCs, an alternative fish dietary bioaccumulation approach will be recommended in the future by OECD TG 305. The dietary approach yields a biomagnification factor (BMF) rather than a BCF. Being able to estimate a $\mathrm{BCF}$ from the data generated in the dietary study would be a great advantage and would meet an accepted regulatory need. Therefore, the estimation of $\mathrm{BCF}_{\mathrm{K}}$ values from data generated in the dietary studies is currently under investigation to keep the results of bioconcentration and biomagnification studies comparable [29]. However, this approach seems to be very complicated and might be even impossible due to the clear differences in the biomagnification and bioconcentration processes. In addition, defined regulatory cutoff criteria for the biomagnification potential of chemical compounds are still missing. Under such circumstances, the estimation of BCFs for highly HOCs in flow-through fish tests might remain essential in the future. SPME is a state-of-the-art technology which delivers results comparable to conventional extraction methods. Freely dissolved concentrations of (highly) HOCs can be obtained. Consequently, unsuitable experimental conditions for flow-through fish tests can be identified. Thus, the reliability of test results and the quality of BCF values for (highly) HOCs can be improved.

\section{Acknowledgements}

This article is based on a report funded by the German Federal Environment Agency under project FKZ-36301240. The authors would like to thank Gunnar Ehrlich and Ulrich Jöhncke (Environmental Protection Agency, Dessau, Germany) for their critical comments on the first draft of the manuscript.

\section{Author details}

${ }^{1}$ Institute of Soil Science and Soil Conservation, Research Centre for BioSystems, Land Use and Nutrition (IFZ) Justus Liebig University Giessen, Heinrich-Buff-Ring 26-32, Giessen, 35392, Germany ${ }^{2}$ Fraunhofer Institute for Molecular Biology and Applied Ecology (IME), Auf dem Aberg 1,

Schmallenberg, 57392, Germany

\section{Authors' contributions}

All authors contributed in equal parts to this publication. All authors read and approved the final manuscript.

\section{Competing interests}

The authors declare that they have no competing interests.

Received: 18 August 2011 Accepted: 23 January 2012

Published: 23 January 2012

\section{References}

1. Regulation (EC) No $1907 / 2006$ of the European Parliament and of the Council of 18 December 2006 concerning the Registration, Evaluation, Authorisation and Restriction of Chemicals (REACH), establishing a European Chemicals Agency, amending Directive 1999/45/EC and repealing Council Regulation (EEC) No 793/93 and Commission Regulation (EC) No 1488/94 as well as Council Directive 76/769/EEC and Commission Directives 91/155/EEC, 93/67/EEC, 93/105/EC and 2000/21/ EC.. , .

2. OECD: OECD Guidelines for the Testing of Chemicals/Section 3: Degradation and Accumulation. Test No. 305: Bioconcentration: Flowthrough Fish Test Updated Guideline. Paris; 1996.

3. Liska I: Fifty years of solid-phase extraction in water analysis-historical development and overview. J Chromatogr A 2000, 885:3-16.

4. Pawliszyn J: Solid Phase Microextraction: Theory and Practice New York: Wiley-VCH; 1997.

5. In Handbook of Solid Phase Microextraction. Edited by: Pawliszyn J. Peking: Chemical Industry Press; 2009:.

6. Alpendurada MF: Solid-phase microextraction: a promising technique for sample preparation in environmental analysis. J Chromatogr A 2000, 889:3-14.

7. Kataoka $\mathrm{H}$ : Current developments and future trends in solid-phase microextraction techniques for pharmaceutical and biomedical analyses. Anal Sci 2011, 27:893-905.

8. Llompart M, Li K, Fingas M: Solid-phase microextraction and headspace solid-phase microextraction for the determination of polychlorinated biphenyls in water samples. Anal Chem 1998, 70:2510-2515.

9. Potter DW, Pawliszyn J: Rapid determination of polyaromatic hydrocarbons and polychlorinated biphenyls in water using solid-phase microextraction and GC/MS. Environ Sci Technol 1994, 28:298-305.

10. Nilsson T, Ferrari R, Facchetti S: Inter-laboratory studies for the validation of solid-phase microextraction for the quantitative analysis of volatile organic compounds in aqueous samples. Anal Chim Acta 1997, 356:113-123.

11. Ferrari R, Nilsson T, Arena R, Arlati P, Bartolucci G, Basla R, Cioni F, Del Carlo G, Dellavedova P, Fattore E, Fungi M, Grote C, Guidotti M, Morgillo S, 
Müller L, Volante M: Inter-laboratory validation of solid-phase microextraction for the determination of triazine herbicides and their degradation products at $\mathrm{ng} / \mathrm{l}$ level in water samples. J Chromatogr A 1998, 795:371-376

12. German standard methods for the examination of water, waste water, and sludge - Jointly determinable substances (group F) - Part 34: Determination of selected plant treatment agents, biocides and breakdown products; Method using gas chromatography (GC-MS) after solidphase micro extraction (SPME) (F 34) (DIN 38407-34:2006-05)..

13. Jonker MTO, van der Heijden SA: Bioconcentration factor hydrophobicity cutoff: an artificial phenomenon reconstructed. Environ Sci Technol 2007, 41:7363-7369

14. Semple KT, Doick KJ, Jones KC, Burauel P, Craven A, Harms H: Defining bioavailability and bioaccessibility of contaminated soil and sediment is complicated. Environ Sci Technol 2004, 38:228A-231A.

15. Arnot JA, Gobas FAPC: A review of bioconcentration factor (BCF) and bioaccumulation factor (BAF) assessments for organic chemicals in aquatic organisms. Environ Rev 2006, 14:257-297.

16. Yabuta H, Fukushima M, Tanaka F, Ichikawa H, Tatsumi K: Solid-phase microextraction for evaluation of partition coefficients of a chlorinated dioxin and hexachlorobenzene into humic substances. Anal Sci 2004, 20:787-791.

17. Ramos EU, Meijer SN, Vaes WHJ, Verhaar HJM, Hermens JLM: Using solidphase microextraction to determine partition coefficients to humic acids and bioavailable concentrations of hydrophobic chemicals. Environ Sci Technol 1998, 32:3430-3435.

18. Grote C, Levsen K, Wünsch G: An automatic analyzer for organic compounds in water based on solid-phase microextraction coupled to gas chromatography. Anal Chem 1999, 71:4513-4518.

19. Chiou CT, Malcolm RL, Brinton TI, Kile DE: Water solubility enhancement of some organic pollutants and pesticides by dissolved humic and fulvic acids. Environ Sci Technol 1986, 20:502-508.

20. Marschner B: Sorption von polyzyklischen aromatischen Kohlenwasserstoffen (PAK) und polychlorierten Biphenylen (PCB) im Boden/Sorption of polycyclic aromatic hydrocarbons (PAH) and polychlorinated biphenyls (PCB) in soil. J Plant Nutr Soil Sci 1999, 162:1-14.

21. Pörschmann J, Kopinke F-D, Pawliszyn J: Solid-phase microextraction for determining the binding state of organic pollutants in contaminated water rich in humic organic matter. J Chromatogr A 1998, 816:159-167.

22. Böhm L, Düring R-A: Partitioning of polycyclic musk compounds in soil and aquatic environment-experimental determination of $\mathrm{K}_{\mathrm{DOC}}$. I Soils Sediments 2010, 10:708-713.

23. Heringa $M B$, Hermens JLM: Measurement of free concentrations using negligible depletion-solid phase microextraction (nd-SPME). Trends Anal Chem 2003, 22:575-587.

24. Leslie HA, ter Laak TL, Busser FJM, Kraak MHS, Hermens JLM: Bioconcentration of organic chemicals: is a solid-phase microextraction fiber a good surrogate for biota? Environ Sci Technol 2002, 36:5399-5404.

25. Reichenberg F, Mayer P: Two complementary sides of bioavailability: accessibility and chemical activity of organic contaminants in sediments and soils. Environ Toxicol Chem 2006, 25:1239-1245.

26. Lu X, Skwarski A, Drake B, Reible DD: Predicting bioavailability of PAHs and PCBs with porewater concentrations measured by solid-phase microextraction fibers. Environ Tox Chem 2011, 30:1109-1116.

27. You J, Harwood AD, Li H, Lydy MJ: Chemical techniques for assessing bioavailability of sediment-associated contaminants: SPME versus Tenax extraction. J Environ Monit 2011, 13:792-800.

28. Böhm L, Schlechtriem C, Düring R-A: Eignung der Festphasenmikroextraktion (SPME) zur Bestimmung von Analytkonzentrationen in der Wasserphase in Biokonzentrationstests. In 4. Gemeinsame Jahrestagung von SETAC GLB und GDCh, September 2010; Dessau. Volume 22. Umweltwiss Schadst Forsch; 2010:267-494.

29. Crookes M, Brooke D: Estimation of Fish Bioconcentration Factor (BCF) from Depuration Data (Environment Agency Science Report) Bristol: Environment Agency; 2011.

doi:10.1186/2190-4715-24-4

Cite this article as: Düring et al: Solid-phase microextraction for bioconcentration studies according to OECD TG 305. Environmental Sciences Europe 2012 24:4.

\section{Submit your manuscript to a SpringerOpen ${ }^{\mathcal{O}}$ journal and benefit from:}

- Convenient online submission

- Rigorous peer review

- Immediate publication on acceptance

- Open access: articles freely available online

- High visibility within the field

- Retaining the copyright to your article

Submit your next manuscript at $\boldsymbol{\nabla}$ springeropen.com 